\title{
Finite-size effects of dimensional crossover in quasi-two- dimensional three-state Potts model
}

\author{
Atsushi Yamagata \\ Department of Physics, Tokyo Institute of Technology, Oh-okayama, Meguro- \\ ku, Tokyo 152, Japan
}

Running title Quasi-two-dimensional three-state Potts model

Keywords Quasi-two-dimensional magnet, Three-state Potts model, Finitesize scaling, Dimensional crossover, Monte Carlo simulation

PACS classification codes 02.70.Lq, 64.60.Cn, 75.10.Hk

\begin{abstract}
A nearest neighbour spin pair of the quasi-two-dimensional three-state Potts model interacts with the strength $J(>0)$ in the $x y$-plane and with $\lambda J(0 \leq$ $\lambda \ll 1)$ in the $z$-axis. The phase transition is of second-order when $\lambda=0$ and is of first-order when $\lambda>0$. The dimensional crossover occurs with a change of the order of the phase transition. We study the finite-size effects of the phenomenon by using a Monte Carlo method with a multi-spin coding technique. The prediction of the finite-size scaling theory is consistent with the Monte Carlo results.
\end{abstract}




\section{Introduction}

A quasi-two-dimensional system is a three-dimensional one in which the ratio $\lambda$ of the interplanar to the intraplanar exchange interactions is small. If the system exhibits a phase transition, we can see a crossover from two-dimensional to three-dimensional behaviour as the critical point is approached. Many authors have studied quasi-two-dimensional antiferromagnets by neutron scattering experiments [1].

The theoretical studies of the dimensional crossover have been carried out by using perturbation theory [2]- [5], high temperature series expansion [6]- [13], generalized homogeneous functions [14], and rigorous approach [15][17]. The results are that the critical and the crossover temperature are singular with respect to $\lambda$. They behave as $\lambda^{1 / \phi}$ where $\phi$ is the crossover exponent [18]. It has been shown that $\phi$ is equal to the critical exponent $\gamma$ for the susceptibility of the system with $\lambda=0$. The present author discussed finite-size scaling and performed Monte Carlo simulaltions of the quasi-twodimensional Ising model for the first time [19].

In the above mentioned researches there was an assumption that the order of the phase transition was unaltered. In this paper we study finite-size scaling of the dimensional crossover in which it changes. We perform Monte Carlo simulations of the three-state ferromagnetic Potts model [20]. The phase transition is of second-order in two dimensions [21] and is of first-order in three dimensions [22]. In the next section we review the finite-size scaling theory for the quasi-two-dimensional systems briefly. The finite-size scaling form of an effective transition temperature is presented. In section 3 we describe an algorithm for the multi-spin coding technique used in our Monte Carlo simulations of the quasi-two-dimensional three-state Potts model. The Monte Carlo data are compared with the prediction of the finite-size scaling theory in section 4 . A summary is given in section 5 .

\section{Finite-size scaling theory}

We review the finite-size scaling theory for the quasi-two-dimensional systems briefly. The detailed discussion is in the reference [19]. Let us consider the three-state Potts model on the simple cubic lattice for the sake of con- 
creteness. The Hamiltonian is

$$
\mathcal{H}_{\lambda}=\sum_{\langle i j\rangle} J_{i j}\left[1-\delta\left(\sigma_{i}, \sigma_{j}\right)\right]+H \sum_{i}\left[1-\delta\left(\sigma_{i}, 1\right)\right]
$$

where $\sigma_{i}$ is a Potts spin variable located $i$ th lattice site and which takes on the value 1,2 , and 3 . The first summation is over all nearest neighbour pairs on the lattice, the second summation over all lattice sites. The strength $J_{i j}$ of the interaction for the nearest neighbour pair $i j$ is $J(>0)$ in the $x y$-plane and $\lambda J(0 \leq \lambda \ll 1)$ in the $z$-axis. $H$ is an external magnetic field. When $\lambda=0$, the equation (国) consists of two-dimensional three-state ferromagnetic Potts models which are independent of each other.

We assume that the free energy per spin measured by $k_{\mathrm{B}} T$, where $k_{\mathrm{B}}$ is Boltzmann's constant and $T$ is the temperature, is a generalized homogeneous function of variables

$$
t_{0}=T / T(0)-1
$$

$h=H / k_{\mathrm{B}} T$, and $\lambda$ as $t_{0}, h, \lambda \rightarrow 0$, where $T(0)$ is the critical temperature of the system with $\lambda=0$. We can derive

$$
f\left(t_{0}, h, \lambda\right)=\left|t_{0}\right|^{2-\alpha} f^{ \pm}\left(h /\left|t_{0}\right|^{\beta+\gamma}, \lambda /\left|t_{0}\right|^{\phi}\right)
$$

with a scaling function $f^{ \pm}(x, y)$ where $+(-)$ refers to $t_{0}>0\left(t_{0}<0\right)$, and $\alpha+2 \beta+\gamma=2$ where $\alpha, \beta$, and $\gamma$ are the critical exponents of the two-dimensional system for the specific heat, the magnetization, and the susceptibility, respectively. The number $\phi$ is the crossover exponent and is equal to $\gamma$. From (2) we get the behaviour of $T(\lambda)$ that is the transition temperature of the system with $\lambda$ as follows.

$$
T(\lambda) / T(0)-1=A^{T} \lambda^{1 / \phi}
$$

where $A^{T}$ is a constant.

Let us consider the three-state Potts model (1) on an $L \times L \times L$ simple cubic lattice to see the finite-size effects [23]-25] of (3). To avoid surface effects we impose periodic boundary conditions. We assume that the free energy per spin is a generalized homogeneous function of $t_{0}, h, \lambda$, and $L$ as $t_{0}, h, \lambda, 1 / L \rightarrow 0$ and the system is characterized by $L / \xi\left(t_{0}\right)$ where $\xi\left(t_{0}\right)$ is 
the correlation length of the system of $h=\lambda=1 / L=0$. Using (2) we can derive

$$
f\left(t_{0}, h, \lambda, L\right)=L^{-(2-\alpha) / \nu} \tilde{f}\left(t_{0} L^{1 / \nu}, h L^{(\beta+\gamma) / \nu}, \lambda L^{\phi / \nu}\right)
$$

where $\tilde{f}(x, y, z)$ is a scaling function and $\nu$ is the critical exponent for $\xi\left(t_{0}\right)$.

Let us define an effective transition temperature as the position, $T_{L}(\lambda)$, of the peak of the specific heat. From (1) we get

$$
T_{L}(\lambda) / T(0)-1=L^{-1 / \nu} \tilde{T}\left(\lambda L^{\phi / \nu}\right)
$$

where $\tilde{T}(x)$ is a scaling function. If $\tilde{T}(x) \rightarrow A^{T} x^{1 / \phi}$ as $x \rightarrow+\infty$, the equation (3) is reproduced in the limit $L \rightarrow+\infty$ for a fixed value of $\lambda(>0)$. The prediction (5) will be compared with the Monte Carlo data in section 4 .

\section{Monte Carlo simulations}

To confirm the prediction (5) of the finite-size scaling theory, we perform Monte Carlo simulations [26, 27] of the quasi-two-dimensional three-state Potts model (11) in $H=0$ on the $L \times L \times L$ simple cubic lattice under fully periodic boundary conditions. We use a multi-spin coding technique [28, 29] to simulate a large number of systems simultaneously. Since the FORTRAN compiler on the HITAC S-820/80 computer, we have used, treats 32-bit integers, we can update 32 systems independently. Three-state Potts spin variables located at identical lattice sites are stored in the 32-bit positions of two words 30, 31.

An algorithm is as follows. Let us consider a flip of a spin $\sigma_{0}$. The change in the energy on flipping the spin, $\sigma_{0} \rightarrow \sigma_{0}^{\prime}$, is

$$
\begin{aligned}
\Delta E / J= & -\sum_{j=1}^{4}\left\{\left[1-\delta\left(\sigma_{0}, \sigma_{j}\right)\right]-\left[1-\delta\left(\sigma_{0}^{\prime}, \sigma_{j}\right)\right]\right\} \\
& -\lambda \sum_{k=5}^{6}\left\{\left[1-\delta\left(\sigma_{0}, \sigma_{k}\right)\right]-\left[1-\delta\left(\sigma_{0}^{\prime}, \sigma_{k}\right)\right]\right\}
\end{aligned}
$$

where $\sigma_{j}(j=1,2,3,4)$ is the nearest neighbour spin of $\sigma_{0}$ in the $x y$-plane and $\sigma_{k}(k=5,6)$ in the $z$-axis. Using variables

$$
n_{x y} \equiv \sum_{j=1}^{4}\left\{\left[1-\delta\left(\sigma_{0}, \sigma_{j}\right)\right]-\left[1-\delta\left(\sigma_{0}^{\prime}, \sigma_{j}\right)\right]+1\right\}
$$


and

$$
n_{z} \equiv \sum_{k=5}^{6}\left\{\left[1-\delta\left(\sigma_{0}, \sigma_{k}\right)\right]-\left[1-\delta\left(\sigma_{0}^{\prime}, \sigma_{k}\right)\right]+1\right\}
$$

we have

$$
-\Delta E / J=n_{x y}+\lambda n_{z}-4-2 \lambda .
$$

Defining a variable $n \equiv 5 n_{x y}+n_{z}+10$, we may see the energy change as a function of $n:(-\Delta E / J)_{n}, n \in\{10,11, \ldots, 54\}$.

Using a random variable $W$ which takes an integer value, we flip the spin if $n+w \geq 32$ where $w$ is a possible value of $W$. The distribution of $W$ is determined by

$$
\begin{aligned}
\operatorname{Prob}\{W \geq 32-n\} & =\min \left(e^{K(-\Delta E / J)_{n}}, 1\right) \\
& = \begin{cases}e^{K(-\Delta E / J)_{n}}, & 10 \leq n<32 \\
1, & 32 \leq n \leq 54\end{cases}
\end{aligned}
$$

where $K=J / k_{\mathrm{B}} T$. Thus the update is accepted with the probability $\min \left(e^{-\beta \Delta E}, 1\right)$ where $\beta=1 / k_{\mathrm{B}} T$. This is the same procedure as of the Metropolis algorithm [32]. We express $n+w(\in\{10,11, \ldots, 76\})$, which can be calculated with logical operations, as the binary notation: $\sum_{l=0}^{6} x_{l} 2^{l}$, $x_{l} \in\{0,1\}$. In our algorithm we carry out the update when $x_{5}=1$ or $x_{6}=1$ although the Metropolis procedure needs to refer to the inequality $r \leq \min \left(e^{-\beta \Delta E}, 1\right)$ where $r$ is a possible value of a random variable $R$ with uniform distribution over $[0,1]$. The algorithm explained here is for $\lambda \in(0,1 / 4)$. It is sufficient to study the system since we are interested in the case of the small value of $\lambda$.

The pseudorandom numbers are generated by the Tausworthe method [33, 34]. We measure physical quantities at a temperature over $10^{5}$ Monte Carlo steps per spin (MCS/spin) after discarding $10^{4} \mathrm{MCS} / \mathrm{spin}$ to attain equilibrium. Physical quantities are calculated by the multi-step bitwise summation algorithm 33, 34. Our algorithm achieves a speed of 25 million spins per second on a $30 \times 30 \times 30$ lattice with measurements at every step. Let us denote the average of a physical quantity, $O$, in each system by $\langle O\rangle_{i}$, $i=1,2, \ldots, 32$. The expectation value is given by

$$
\overline{\langle O\rangle}=\frac{1}{32} \sum_{i=1}^{32}\langle O\rangle_{i}
$$


the standard deviation by

$$
\Delta\langle O\rangle=\left(\overline{\langle O\rangle^{2}}-{\overline{\langle O\rangle^{2}}}^{2}\right)^{1 / 2} / \sqrt{31}
$$

\section{Monte Carlo results}

In the reference 19 we reported that there was hysteresis in magnetic quantities (magnetization, susceptibility, ... ) but we could not see it in the energy, the specific heat:

$$
C=k_{\mathrm{B}} \beta^{2}\left(\overline{\left\langle\mathcal{H}_{\lambda}^{2}\right\rangle}-\overline{\left\langle\mathcal{H}_{\lambda}\right\rangle^{2}}\right) / L^{3},
$$

and the fourth-order cumulant of the energy for the small value of $\lambda$ or a small number of MCS/spin. The hysteresis vanished for the large value of $\lambda$ or a large number of MCS/spin. We have observed similar behaviour for the quasi-two-dimensional three-state Potts model. According to [19], we analyse the peak position, $T_{L}(\lambda)$, of the specific heat since we can obtain useful information about phase transitions from it.

Figure 1 shows the temperature dependence of $C$ of $L=20$ system for various $\lambda$. The solid curves are obtained by the smoothing procedure of the fourth-order $B$-spline [35]. As $\lambda$ increases, the shape of the curve becomes sharper and the peak position $T_{20}(\lambda)$ becomes higher. Then we get the peak height and $T_{20}(\lambda)$.

Figure 2 shows the finite-size scaling plot of $T_{L}(\lambda)$. We have used the exact values of the critical temperature, $k_{\mathrm{B}} T(0) / J=1 / \ln (1+\sqrt{3})$, and the critical exponents, $\phi=\gamma=13 / 9$ and $\nu=5 / 6$, of the two-dimensional threestate ferromagnetic Potts model 20]. The data fall on a common curve. It is consistent with (5). The slope seems to approach $1 / \phi=1 / \gamma=9 / 13$. It indicates that the asymptotic behaviour of the finite-size scaling function is $\tilde{T}(x) \rightarrow$ const. $\times x^{1 / \phi}$ as $x \rightarrow+\infty$.

\section{Summary}

The dimensional crossover occurs with a change of the order of the phase transition in the quasi-two-dimensional three-state Potts model. We have studied the finite-size effects of the phenomenon by using a Monte Carlo method. An algorithm of a multi-spin coding technique for this model has 
been presented. The prediction (5) of the finite-size scaling theory has been consistent with the Monte Carlo results. We have seen the asymptotic behaviour of the finite-size scaling function on the system with $\lambda \leq 0.24$ and $L \leq 30$.

\section{Acknowledgements}

The author would like to thank Dr. Katsumi Kasono for useful discussions and critical reading of the manuscript. We performed the simulations on the HITAC S-820/80 computer of the Computer Centre, Institute for Molecular Science, Okazaki National Research Institutes and of the Computer Centre at Hokkaido University. This study was supported by a Grant-in-Aid for Scientific Research from the Ministry of Education, Science, and Culture, Japan. 


\section{References}

[1] M.F. Collins, Magnetic Critical Scattering (New York, Oxford, 1989) p. 63.

[2] E.I. Nesis, Soviet Phys.-Solid State 7 (1965) 534.

[3] R. Abe, Prog. Theor. Phys. 44 (1970) 339.

[4] M. Suzuki, Prog. Theor. Phys. 46 (1971) 1054.

[5] A. Coniglio, Physica 58 (1972) 489.

[6] G. Paul and H.E. Stanley, Phys. Lett. A 37 (1971) 347.

[7] G. Paul and H.E. Stanley, Phys. Rev. B 5 (1972) 2578.

[8] J. Oitmaa and I.G. Enting, Phys. Lett. A 36 (1971) 91.

[9] D.C. Rapaport, Phys. Lett. A 37 (1971) 407.

[10] F. Harbus, R. Krasnow, L.L. Liu and H.E. Stanley, Phys. Lett. A 42 (1972) 65.

[11] F. Harbus and H.E. Stanley, Phys. Rev. B 7 (1973) 365.

[12] F. Harbus and H.E. Stanley, Phys. Rev. B 8 (1973) 2268.

[13] R. Krasnow, F. Harbus, L.L. Liu and H.E. Stanley, Phys. Rev. B 7 (1973) 370 .

[14] A. Hankey and H.E. Stanley, Phys. Rev. B 6 (1972) 3515.

[15] L.L. Liu and H.E. Stanley, Phys. Rev. Lett. 29 (1972) 927.

[16] L.L. Liu and H.E. Stanley, Phys. Rev. B 8 (1973) 2279.

[17] C.A.W. Citteur and P.W. Kasteleyn, Phys. Lett. A 42 (1972) 143.

[18] E. Riedel and F. Wegner, Z. Phys. 225 (1969) 195.

[19] A. Yamagata, Physica A 205 (1994) 665. 
[20] F.Y. Wu, Rev. Mod. Phys. 54 (1982) 235, erratum: ibid. 55 (1983) 315.

[21] R.J. Baxter, J. Phys. C 6 (1973) L445.

[22] A. Yamagata, J. Phys. A 26 (1993) 2091, and references therein.

[23] M.E. Fisher, Critical Phenomena, Proc. 1970 Enrico Fermi Summer School, vol. 51, M.S. Green, ed. (Academic Press, New York, 1970) p. 1.

[24] M.N. Barber, Phase Transitions and Critical Phenomena, vol. 8, C. Domb and J.L. Lebowitz, eds. (Academic Press, London, 1983) p. 145 .

[25] V. Privman, Finite Size Scaling and Numerical Simulation of Statistical Systems, V. Privman, ed. (World Scientific, Singapole, 1990) p. 1.

[26] K. Binder, Monte Carlo Methods in Statistical Physics, K. Binder, ed. (Springer, Berlin, 1979) p. 1.

[27] K. Binder and D. Stauffer, Applications of the Monte Carlo Method in Statistical Physics, 2nd ed., K. Binder, ed. (Springer, Berlin, 1987) p. 1.

[28] G. Bhanot, D. Duke and R. Salvador, Phys. Rev. B 33 (1986) 7841.

[29] G. Bhanot, D. Duke and R. Salvador, J. Stat. Phys. 44 (1986) 985.

[30] M. Kikuchi and Y. Okabe, J. Phys. Soc. Jpn. 61 (1992) 3503

[31] Y. Okabe and M. Kikuchi, Computational Approaches in CondencedMatter Physics, S. Miyashita, M. Imada and H. Takayama, eds. (Springer, Berlin, 1992) p. 193.

[32] N. Metropolis, A.W. Rosenbluth, M.N. Rosenbluth, A.H. Teller and E. Teller, J. Chem. Phys. 21 (1953) 1087.

[33] N. Ito and Y. Kanada, Supercomputer 5 (1988) 31.

[34] N. Ito and Y. Kanada, Proceedings of Supercomputing '90 (IEEE Computer Society Press, Los Alamitos, 1990) p. 753.

[35] T. Tsuda, Suchi Shori Programming (Iwanami, Tokyo, 1988) p. 151. 


\section{Figure captions}

Figure 1 Temperature $\left(K=J / k_{\mathrm{B}} T\right)$ dependence of the specific heat of the $L=20$ system for various $\lambda: 0.04,0.08,0.1,0.12,0.14,0.16,0.18,0.20$, $0.22,0.24$. The solid curves are obtained by the smoothing procedure of the fourth-order $B$-spline. As $\lambda$ increases, the shape of the curve is sharper and $T_{20}(\lambda)$ becomes higher.

Figure 2 Finite-size scaling plot of the effective transition temperature. The data of the system with $L=10,20$, and 30 are denoted by $\bigcirc, \times$, and $\square$, respectively. We have set that $k_{\mathrm{B}} T(0) / J=1 / \ln (1+\sqrt{3}), \phi=\gamma=13 / 9$, and $\nu=5 / 6$. The data fall on a common curve. The solid line shows that $(9 / 13) \ln \left(\lambda L^{26 / 15}\right)$. Errors are less than the symbol size. 


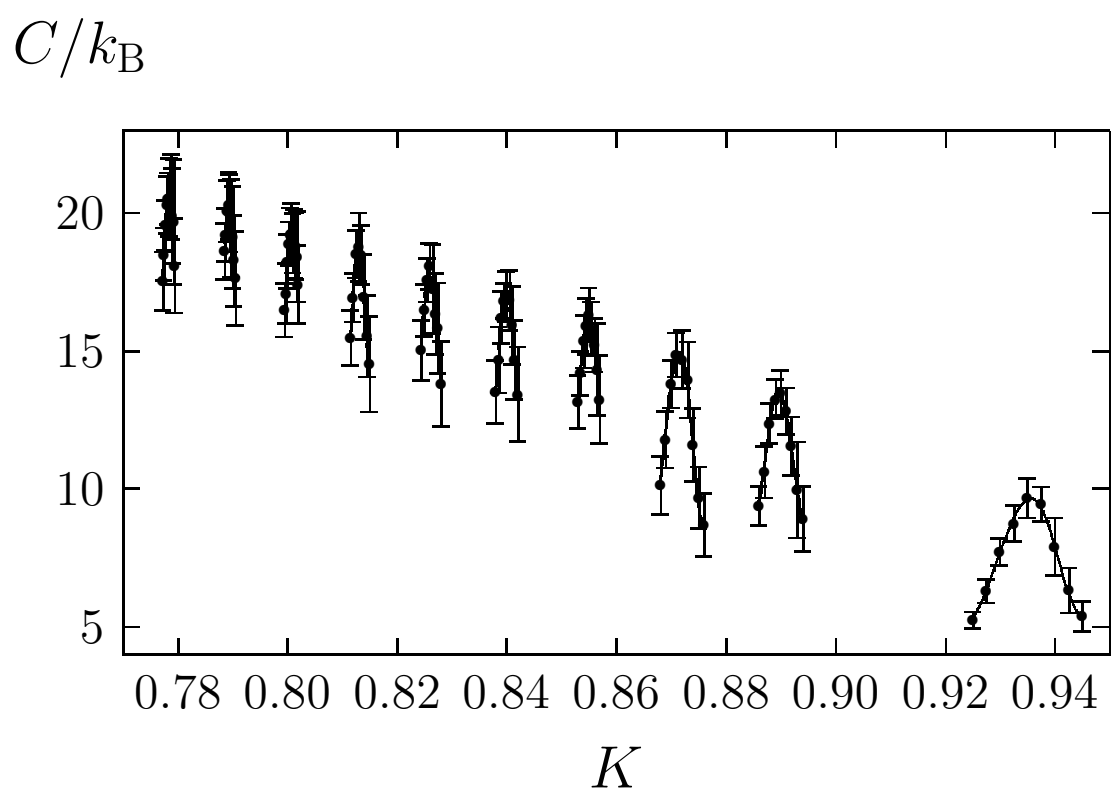

Figure 1

Atsushi Yamagata 


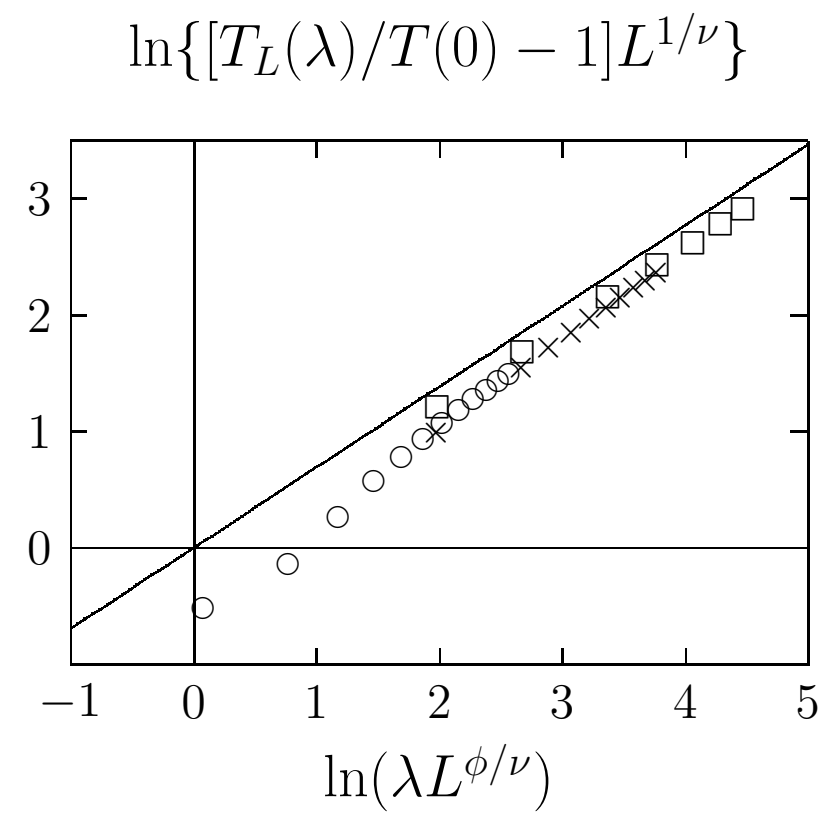

Figure 2

Atsushi Yamagata 